\title{
Heart transplantation
}

\section{Background}

Heart transplantation is a major operation whereby a recipient's heart is replaced by that of a deceased donor. While eligibility varies internationally, heart transplantations are generally reserved for individuals with advanced heart failure when maximal alternative treatment options have failed. Advanced heart failure occurs when the heart fails to pump sufficient blood to meet the demands of the body's tissues and organs. This often causes debilitating symptoms, such as severe fatigue, leg swelling, and shortness of breath. Possible causes of heart failure include coronary artery disease, valve disease, congenital heart disease, rhythm disorders, and cardiomyopathy, whereby the muscles of the heart have been damaged. The process of heart transplantation requires thorough screening and assessment of donors and recipients prior to surgery. After the operation, close monitoring and extensive rehabilitation is required to maximize the likelihood of clinical benefits.

\section{Indications and eligfibility}

Indications and eligibility for heart transplantation differ between institutions and often require a multi-disciplinary medical team for a thorough assessment to ensure appropriate allocation of limited donor organs. Most transplant recipient candidates have had chronic heart failure for a number of years, but selected individuals with acute heart failure may be offered transplantation as the only option to prolong survival. There are a number of factors that may exclude an individual from receiving a heart transplant due to an increased risk of death from the operation or a decreased likelihood of recovering after the operation. These include active cancer, diabetes, obesity, infections, substance abuse and an inability to comply with the medication regime or rehabilitation process following surgery. Assessment for the donor includes investigations to ensure adequate function of the heart, screening for infectious diseases such as HIV, hepatitis B and C, as well as active cancer. Other factors that influence the donor-recipient matching process include their age, heart size, distance apart, and immunological matching.

\section{Operation}

Heart transplantation requires an incision through the sternum, or breastbone, and the use of a cardiopulmonary bypass machine that functions as the patient's heart and lungs during the operation. The donor heart is removed or 'retrieved' by the surgical team after a complete assessment to ensure its adequate function. Meanwhile, the recipient has his/her heart removed whilst on cardiopulmonary bypass and the surgeon connects the donor heart onto the recipient's major vessels upon arrival of the donor heart.

\section{Benefits}

For individuals with end-stage heart failure, heart transplantation may prolong survival by approximately 10 years following surgery. Close to $85 \%$ of individuals survive the first year after heart transplantation. These patients may also improve their symptoms of heart failure, with a more active lifestyle and improved quality of life.

\section{Risks}

As with all major heart operations, heart transplantation carries potential risks for bleeding, infection, heart attack, stroke, blood clots, and death. Following the operation, there is a need for lifelong immune-suppressing medications that prevent rejection of the donor heart by the recipient's immune system, which recognizes the donor heart as being 'foreign'. These medications that suppress the immune system may in themselves cause an increased risk of infections and cancers. The most common complications in the year following heart transplantation include graft failure, rejection and infection. Complications after the first year include cancers and a condition called 'cardiac allograft vasculopathy', whereby the donor heart's arteries thicken, possibly leading to a heart attack. The benefits and risks of heart transplant must be weighed against each other and discussed with your surgeon and a multi-disciplinary transplant team.

For more information, please visit the following websites: https://www.mayoclinic.org/tests-procedures/heart-transplant/ about/pac-20384750

https://my.clevelandclinic.org/departments/transplant/ programs/heart/process

https://www.heartfoundation.org.au/images/uploads/ publications/Heart-Transplants-Donations.pdf

doi: 10.21037/acs.2018.01.11

Section Writer: Christopher Harris, Christopher Cao

Illustration Editor: Beth Croce

Section Editor: Stine Munkholm-Larsen
For specific information concerning your medical condition, ACS suggests that you consult your physician. This page may be photocopied non-commercially by physicians to share with patients. 